\title{
EXPLORING CULTURAL HERITAGE RESOURCES IN A 3D COLLABORATIVE ENVIRONMENT
}

\author{
A. Respaldiza ${ }^{\text {a, }}$, M. Wachowicz ${ }^{\text {b }}$, A. Vázquez Hoehne ${ }^{\text {a }}$ \\ ${ }^{a}$ Dept. of Surveying Engineering and Cartography, Technical University of Madrid, UPM, Autovía de Valencia km 7,5, \\ E-28031 Madrid, Spain - (ma.respaldiza, antonio.vazquez.hoehne)@upm.es \\ ${ }^{\mathrm{b}}$ Dept. of Geodesy and Geomatics Engineering, University of New Brunswick, 15 Dineen Drive, Fredericton NB, E3B \\ 5A3 Canada - monicaw@unb.ca
}

KEY WORDS: Information Visualization, Metadata, Cultural Heritage, 3D Collaborative Environment

\begin{abstract}
:
Cultural heritage is a complex and diverse concept, which brings together a wide domain of information. Resources linked to a cultural heritage site may consist of physical artefacts, books, works of art, pictures, historical maps, aerial photographs, archaeological surveys and 3D models. Moreover, all these resources are listed and described by a set of a variety of metadata specifications that allow their online search and consultation on the most basic characteristics of them. Some examples include Norma ISO 19115, Dublin Core, AAT, CDWA, CCO, DACS, MARC, MoReq, MODS, MuseumDat, TGN, SPECTRUM, VRA Core and Z39.50. Gateways are in place to fit in these metadata standards into those used in a SDI (ISO 19115 or INSPIRE), but substantial work still remains to be done for the complete incorporation of cultural heritage information. Therefore, the aim of this paper is to demonstrate how the complexity of cultural heritage resources can be dealt with by a visual exploration of their metadata within a 3D collaborative environment. The 3D collaborative environments are promising tools that represent the new frontier of our capacity of learning, understanding, communicating and transmitting culture.
\end{abstract}

\section{INTRODUCTION}

Cultural heritage is a complex and diverse concept, which brings together a wide domain of information. Resources linked to a cultural heritage site may consist of physical artefacts, books, works of art, pictures, historical maps, aerial photographs, archaeological surveys and 3D models. Moreover, all these resources are listed and described by a set of a variety of metadata specifications that allow their online search and consultation on the most basic characteristics of them. Some examples include Norma ISO 19115, Dublin Core, AAT, CDWA, CCO, DACS, MARC, MoReq, MODS, MuseumDat, TGN, SPECTRUM, VRA Core and Z39.50. Gateways are in place to fit in these metadata standards into those used in a SDI (ISO 19115 or INSPIRE), but substantial work still remains to be done for the complete incorporation of cultural heritage information. The ArchaeoLandscapes Europe project (ArcLand) is a particular effort towards making better use of existing archaeological, LIDAR and photogrammetric survey data and their respective metadata specifications for sharing cultural heritage information which is often scattered all over Europe (see http://www.archaeolandscapes.eu/ for more details). The ARENA portal has now been running since 2004 under the auspices of both the DARIAH project and the ACE project. A second phase ARENA 2 is planned to take full advantage of web services and a SOA approach whilst demonstrating that 'legacy' systems (such as Z39.50) can be integrated into a SDI architecture (see http://ads.ahds.ac.uk/arena/ for more details). They are working on various proposals for creating domains pecific standards such as 3D Conform EPOCH (http://www.3dcoform.eu/ and http://www.epoch.eu/).
In addition to research focused on building environments to support collaborative work with cultural heritage information, attention is beginning to be directed to human aspects of collaboration asynchronous at a distance. A starting point for supporting different-place geocollaboration is provided by the development of web and technologies from distributed databases, tools together with a Spatial Data Infrastructure; these developments were reviewed for MacEachren (MacEachren, 2001; MacEachren et al., 1999, 2005, 2006; Pike et al., 2005), Balram and Dragićević (Balram and Dragićević, 2006; Dragićević, 2004; Dragićević and Balram, 2004) and, Gong and Lin (Gong and Lin, 2006). This work has considered both metadata and interface issues for serving the cultural heritage information through the web, mainly those concerned with how to visually represent the metadata to users.

Currently we can count few metadata of applications of 3D virtual reconstructions in cultural heritage and in computer graphics. The metadata reconstruction is in the capacity of showing the spatial-temporal, semantic, symbolic and interpretative relations between the model, the final result, and the interpretation process. The aim of this research is to experiment a multi-user domain on the web aimed to a multidisciplinary scientific community: historians, archivist, archaeologists, experts in human and social sciences, communication experts. These developments were reviewed for Forte (Forte, 1997, 2000, 2003; Forte and Pietroni, 2006). Different hypotheses corresponding to the "possible realities" can coexist, showing the reconstruction of the past. All cultural heritage information converge in a virtual scenario on the web where the scientific community can meet and interact in real time, exchange and test hypothesis, share data and simulate

* Arantza Respaldiza ma.respaldiza@upm.es 
different scenarios in order to discuss possible interpretations and methods. The envisaged virtual space will be an editable and dynamic environment in continuous evolution and able to be updated with new information.

Therefore, the aim of this paper is to demonstrate how the complexity of cultural heritage resources can be dealt with by a visual exploration of their metadata within a $3 \mathrm{D}$ collaborative environment. Towards this end, a metadata visualisation approach is proposed for creating a formal structure for an implicit and explicit representation of the connections and voids between different current domain-specific standards. The case study is Risk Map for Tossa de Mar (Girona, Spain) was used for the implementation. The Risk Map characterizes the presence and territorial diffusion of the historic, cultural and environmental heritage and values its vulnerability. Furthermore the Risk Map observes, describes and values dangerous levels present in the territory and pertinent static-structural, ambient air and anthropic dimensions.

\section{METHODOLOGY}

Cultural heritage information as a representation of the real world involves three different levels of representation. There are the conceptual representation, digital representation, and visual representation (Ahonen-Rainio, 2005; Laurini and Thompson, 1992; Worboys, 1995), and all of concern to a user of metadata. We propose a 3D modelling metadata structure based on the previous work carried out by Styliadis (Styliadis et al., 2009). First the data forming the basis of the modelling study are examined as primary metadata, then the principles of both the analysis and the restitution are presented (secondary and tertiary metadata), and finally the hypothesis formulation (fourth level metadata) and following the reconstruction hypothesis formulation (fifth level metadata) are documented.

Moreover, this 3D modelling metadata structure is combined with a metadata visualisation approach as proposed by Riley (Riley, 2010) and Fairbairn (Fairbairn et al., 2001). As a result, visual representations are used for creating hierarchies among the metadata specifications of cultural heritage resources and their different interpretations, as well as their dependencies.

The aspects of visualization research (Fairbairn et al., 2001) are mutually interrelated as: data, purpose, technology, impact and form. The five sections identify multiple aspect of the relationship between representation and visualization.

2.1 Data: The nature of what kind of data and phenomena are to be represented, the form of representation chosen, for example, conceptual model or database representation.

2.2 Purpuse: The purpose for which representation is undertaken and used, the users for whom representation is undertaken, and the methods and the technologies that enable representation to be accomplished.

2.3 Technology: The changing technology to support new forms of representation, how representations can be accessed and enhanced.

2.4 Impact: The impact of representation form on both understanding and task outcomes, in particular, user interactionwith dynamic representations and with other users.
2.5 Form: The representation purpose include matching the representation with generic or specific data handling tasks. And appearance and form of representation, visual design and the user interface.

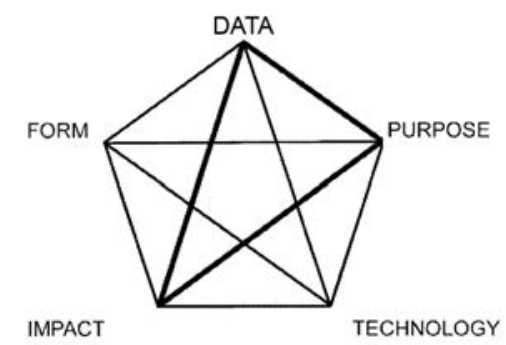

Figure 1. Aspects of visualization research

The issues of concern in visualization research include characteristics of data to be visualized, purpose and form of representation, impact of form on understanding and task outcomes, and technology to support new form of representation. To reiterate, we consider the most critical issues to be those concerned with the characteristics of the data to be handled, including issues of its generalisation, organisation and its inherent attributes.

\section{IMPLEMENTATION}

The implementation was carried out in Improvise (Weaver, 2006). Improvise is a fully-implemented Java software architecture and user interface that enables users to build and browse highly-coordinated visualizations interactively. Users gain precise control over how navigation and selection affects the appearance of data across multiple views. By coupling a shared-object coordination model with a declarative visual query language. And using a potentially infinite number of variations on well-known coordination patterns such as synchronized scrolling, overview and detail, brushing, drill down, and semantic zoom.

In the interface implementation attention has been directed, particularly, toward making the cultural heritage information accessible to a community of users with the metadata visualization (Figure 2). Another components of this work will involve by supporting the collection of locally generated information and comparing public use of a virtual decision making environment (Figures 3 and 4).

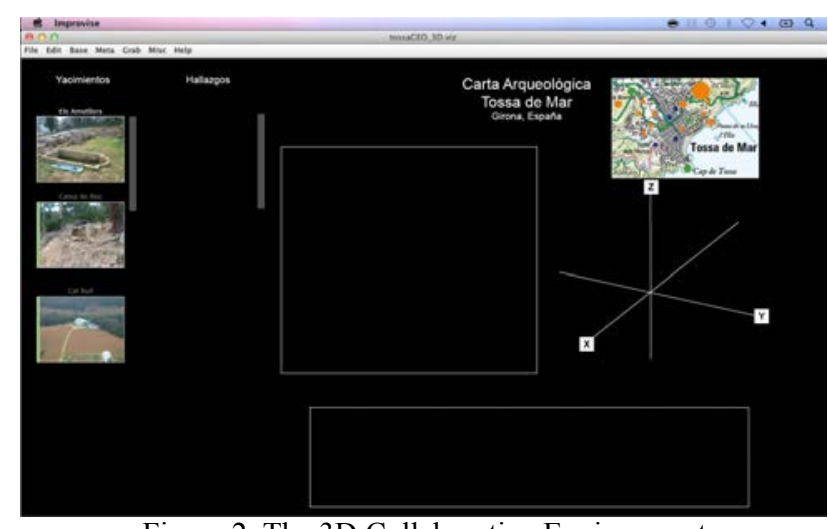

Figure 2. The 3D Collaborative Environment 


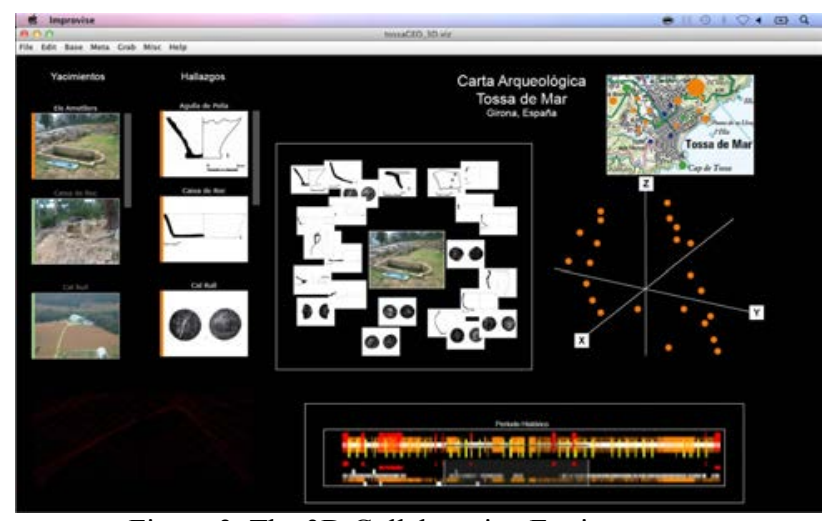

Figure 3. The 3D Collaborative Environment: Archaeological site selection: various resources

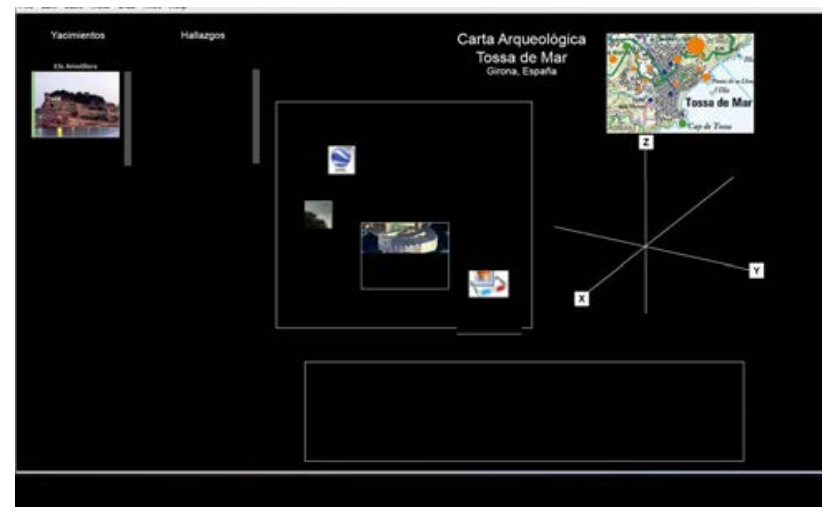

Figure 4. The 3D Collaborative Environment: Archaeological site selection, 3D resources

\section{CONCLUSIONS AND FUTURE WORK}

Collaborative environments have a profound impact on society since they represent a new way of improving user involvement in sharing cultural heritage information. This research work has shown the need for the development of search interfaces that are tailored to the visualization of metadata information accordingly to the different levels of collaboration among users due to their forms of collaboration, channels of communication, and use of cultural heritage information. The 3D collaborative environments are promising tools that represent the new frontier of our capacity of learning, understanding, communicating and transmitting culture.

Further research is needed to study the social behaviour of users within the 3D collaborative environment: analyse them interacting in $3 \mathrm{D}$ with cultural heritage information, constructing cyber maps and landscapes. A possible scenario is the $3 \mathrm{D}$ collaborative environment where the users can interact in 3D, discussing about key features of the information, interpretations and general overviews. Therefore, our future research work will be focussed on the validation of the interpretative and reconstructive process and sharing of all the activities with the community.

\section{ACKNOWLEDGEMENTS}

This work was funded by España Virtual CENIT project funded by the CDTI in the program through 2010 Ingenio CNIG.

\section{REFERENCES}

Ahonen-Rainio, P., 2005. Visualization of Geospactial Metadata for Selecting Geographic Datasets. Helsinki University of Technology. February.

Balram, S. and Dragićević, S., 2006. Collaborative Geographic Information Systems and Science: A Transdisciplinary Evolution. In: Collaborative Geographic Information Systems, pp. 341-350.

Balram, S. and Dragićević, S., 2006. Collaborative Geographic Information Systems and Science: Origins, Boundaries, and Structures. In: Collaborative Geographic Information Systems, pp. 1- 22.

Dragićević, S., 2004. The potential of Web-based GIS. Journal of Geographical Systems, 6, pp. 79-81.

Dragićević, S. and Balram S., 2004. A Web GIS collaborative framework to structure and manage distributed planning processes. Journal of Geographical Systems, 6, pp. 133-153.

Fairbairn, D., Andrienko, G., Andrienko, N., Nuziek, G. and Dykes, J., 2001. Representation and its Relationship with Cartographic Visualization. Cartography and Geographic Information Science, 28(1), pp. 13-28.

Forte, M., 1997. Virtual Archaeology - Great Discoveries Brought to Life Through Virtual Reality, London, Thames \& Hudson.

Forte, M., 2000. About virtual archaeology: disorders, cognitive interactions and virtuality. In: Virtual Reality in Archaeology, Oxford, BAR International Series 843, pp. 247-259.

Forte, M., 2003. Mindscape: ecological thinking, cyberanthropology and virtual archaeological landscapes. The reconstruction of archaeological landscapes through digital technologies. In: Proceedings of the First Italy - USA Workshop (Boston, Nov 1-3 2001), vol. 1151, BAR Interntional Series. pp. 95- 108. Oxford: BAR.

Forte M. and Pietroni E., 2006. The Museum's mind: a cybermap for cultural exhibitions. International Journal of Architectural Computing, 01(7), pp. 57-76.

Gong, J. and Lin, H., 2006. A Collaborative Virtual Geographic Environment: Design and Development. In: Collaborative Geographic Information Systems, pp. 186-206.

Laurini, R. and Thompson, D., 1992. Fundamentals of Spatial Information Systems. Academic Press, London.

MacEachren, A.M., 2001. Cartography and GIS: Extending collaborative tools to support virtual teams. Progress in Human Geography, 25(3), pp. 431-444.

MacEachren, A. M., Cai, G., Sharma, R., Rauschert, I., Brewer, I., Bolelli, L., Shaparenko, B., Fuhrmann, S. and Wang, H., 2005. Enabling collaborative geoinformation access and decision-making through a natural, multimodal interface. International Journal of Gegraphical Information Science, 19(3), pp. 293-317.

MacEachren, A., Edsall, R., Haug, D., Baxter, R., Otto, G., Masters, R., Fuhrmann, S. and Qian, L., 1999. Exploring the potential of virtual environments for geographic visualization. In: Proceedings of 95th Annual Meeting, Association of American Geographers, Honolulu, HI. 
MacEachren, A. M., Pike, W., Yu, C., Brewer, I., Weaver, S., Gahegan, M. and Yarnal, B., 2006. Building a GeoColloaboratory: Supporting Human-Environment Regional Observatory (HERO) Collaborative Science Activities. Computers, Environment and Urban Systems, 30(2), pp.: 201225.

Pike, W., Yarnal, B., MacEachren, A., Gahegan, M. and Yu, C., 2005. Infrastructure for human-environment collaboration: Building a prototype for the future of science. Environment, 47(2), pp. 8-21.

Riley, J., 2010. Seeing standards: A visualization of the metadata universe.

http://www.dlib.indiana.edu/ jenlrile/metadatamap (23 Mar. 2012).

Styliadis, A.D., Akbaylar, I.I., Papadopoulou, D.A., Hasanagas, N.D., Roussa, S.A. and Sexidis L.A., 2009. Metadata-based heritage sites modeling with e-learning functionality. Journal of Cultural Heritage, 10, pp. 296-312.

Weaver, C., 2006. Improvise: A User Interface for Interactive Construction of Highly-Coordinated Visualizations. University of Wisconsin-Madison, June.

Worboys, M.F., 1995. GIS: A Computing Perspective. Taylor \& Francis, London. 